\title{
Incidence of sciatic nerve palsy associated with reconstruction plate fixation of posterior wall and posterior column of acetabulum through posterior approach and its prognosis
}

\section{Introduction}

Fractures of the posterior wall of the acetabulum comprise onefourth to one-third of all acetabular fractures and represent the most common pattern of fracture of the acetabulum. It is distinguished from other forms of acetabular fractures. There is usually an associated hip dislocation causing sciatic nerve palsy. Though conservative treatment can be recommended for the stable joint with a small fragment, open reduction and internal fixation are usually needed when the hip was tested to be unstable or a large portion of the posterior wall is involved. ${ }^{1}$ However, several studies demonstrated a significant complication rate after open reduction and internal fixation of posterior-wall fractures including sciatic nerve palsy, even when compared with other forms of acetabular fracture.

The Kocher-Langenbeck (K-L) approach is the most commonly used surgical exposure for the stabilization of acetabular fractures involving a displaced posterior wall. ${ }^{2}$ This type of approach is almost similar to the posterior approach of the hip in hip arthroplasty surgery (Southern Moore approach ). Many of the studies that relate sciatic nerve palsy after posterior approach to the hip were made based on cases where total hip replacement was done and very few were made in cases with posterior acetabular wall reconstruction.

Review of literature reveals the prevalence of perioperative or postoperative sciatic nerve palsy on fixation of posterior wall of acetabulum to be approximately between $5 \%$ to $15 \% .{ }^{3}$ has reported the incidence of postoperative sciatic nerve injury to be at $5 \%$ in his retrospective review on 103 patients with posterior wall fixation through posterior approach. ${ }^{3}$ In a series of studies it was also found out that all patients with a posttraumatic sciatic nerve injury had fracture patterns that had been associated with posterior wall fracture. ${ }^{3}$

Sciatic nerve palsy associated with acetabular fractures may result from:-

(1) Damage at the time of trauma

(2) Iatrogenic injury during reconstructive surgery through posterior approach, or

(3) A late complication of surgery most commonly associated with heterotrophic ossification

Iatrogenic causes have been reported to be due to intraoperative positioning and placement of retractors, positioning of instruments, or placement of implants. It was also reported to have been resulted from several factors in posterior approaches including excessive retraction of the posterior gluteal muscle mass with the hip in flexion or continuous extension of the ipsilateral knee which can result in a stretch injury to the sciatic nerve. ${ }^{4}$

\author{
Volume 10 Issue 6 - 2018 \\ Kamarul Izham Kamarudin, Mohd Hazim \\ Abd Hamid, Siti Sara Yaacob, Denesh Kumar \\ Shunmugam, Suresh Chopra, Abdul Rauf \\ Ahmad \\ Department of Orthopaedic, Malaysia
}

Correspondence: Kamarul Izham Kamarudin, Orthopaedic Specialist, Department of Orthopaedic, Hospital Sultanah Bahiyyah, KM 6 Jalan Langgar, 05460 Alor Setar, Kedah, Malaysia, Tel+6047406233, Email drizham75@gmail.com

Received: April 16, 2018 | Published: November 12, 2018

Several preventive measures have been suggested to prevent injury to the sciatic nerve intraoperatively which include mainly by avoiding iatrogenic stretch injury to the nerve. This was achieved by keeping the hip in extended position and knee flexed during surgery. Intraoperatively, retractors should be carefully placed and extensive posterior retraction with the hip in flexion should be avoided. Instrument or implant placement should be performed safely with the assistance of intraoperative fluoroscopy if necessary. Sometimes the use of somatosensory evoked potentials (SSEP) and spontaneous electromyography (EMG) are beneficial to alert the surgeon to potential sciatic nerve injury intraoperatively.

A hematoma also has been reported to be one of the causes of early post-operative sciatic nerve palsy which in this case the hematoma itself progressively compress the nerve postoperatively resulting in sciatic neuropathy in the early postoperative period. ${ }^{5}$ Heterotopic ossification around the sciatic nerve has also been reported to be another common postoperative cause of sciatic neuropathy but the neuropathy in these cases often presents in a delayed progressive fashion and coincides with the formation of the ectopic bone. ${ }^{6}$ It was found Intraoperatively that, when surgical exploration was made, the sciatic nerve was encased in heterotopic bone and could only be identified distal to the new bone that was formed. ${ }^{7}$

In a retrospective review of 223 sciatic nerve injuries in posterior approach to the hip in hip arthroplasty surgery found that $41 \%$ had a complete or essentially complete recovery, $44 \%$ had a mild persistent deficit, and $15 \%$ had a poor outcome characterized by weakness that limited ambulation and/or persistent dysesthesias. ${ }^{8}$ Pekkarinen and coworkers ${ }^{9}$ reported on 27 sciatic nerve injuries in which $29 \%$ had full recovery, $25 \%$ had fair or partial recovery, and $44 \%$ had considerable disability. In the author's experience, all patients who had complete 
Table Continued...

recovery of function had it by 2 years. ${ }^{10}$ The prognosis for neurologic recovery was related to the degree of nerve damage. All patients who retained some motor function immediately after the operation, or recovered some motor function within 2 weeks of surgery, had a good recovery. Poor prognostic signs include the presence of painful dysesthesia and complete motor and sensory loss with no return within the first 2 weeks

\section{Materials and method}

This retrospective study was conducted in Hospital Sultanah Bahiyyah, Alor Setar and Hospital Sultan Abdul Halim, Sungai Petani. All patients that underwent posterior approach surgery to the acetabulum for acute fracture of posterior wall, fracture of posterior column and transverse fracture of acetabulum in Hospital Sultanah Bahiyyah Alor Setar and Hospital Sultan Abdul Halim Sungai Petani from January 2008 to January 2016 will be collected. Patients who has old fracture of the acetabulum who required complex surgical procedure, who had history of sciatic nerve palsy preoperatively due to posterior hip dislocation and who had undergone revision surgery for the acetabular fracture through posterior approach were excluded from the study. The demographic data that were included in this study were the age of the patient, the sex of the patient, race, sidedness whether it involved right or left side and patern of the acetabular fracture. Patients history and documentations were traced through EHIS system. Preoperative anteroposterior view of the pelvic and affected acetabulum was collected as well as obturator oblique and iliac oblique view (Judet view) was taken to establish diagnosis. Post operative anteroposterior and judet views of the affected acetabulum were also reviewed to identify the type of surgery and the approach made to fix the fractured acetabulum. Documentations on evidence of patients' inability to dorsiflex their big toes and ankle were recorded from the EHIS system.

\section{Results}

(Tables 1-3).

Table I Descriptive statistics of study participants $(n=68)$

\begin{tabular}{ll}
\hline Variables & Frequency (\%) \\
\hline Age (years) & $38.64(16.17 \%)^{*}$ \\
Sex & \\
Female & $12(16.2 \%)$ \\
Male & $56(82.4 \%)$ \\
Race & \\
Malay & $55(79.4 \%)$ \\
Chinese & $7(10.3 \%)$ \\
Indian & $6(8.8 \%)$ \\
Side & \\
Left & $27(39.7 \%)$ \\
Right & $41(60.3 \%)$ \\
Types & \\
Closed transverse fracture of left acetabulum & $3(4.4 \%)$ \\
Closed transverse fracture of right acetabulum & $1(1.5 \%)$ \\
\hline
\end{tabular}

\begin{tabular}{ll} 
Variables & Frequency (\%) \\
\hline Posterior column of left acetabular fracture & $17(25.0 \%)$ \\
Posterior column of right acetabulum fracture & $19(27.9 \%)$ \\
Posterior wall of left acetabulum fracture & $28(41.2)$ \\
Types of operation & \\
Plating of left acetabulum & $25(36.8 \%)$ \\
Plating of right acetabulum & $43(63.2 \%)$ \\
Foot drop & \\
None & $60(88.2 \%)$ \\
Yes & $8(11.8 \%)$ \\
Recovery(n=8) & \\
No & $1(12.5 \%)$ \\
Yes & $7(87.5 \%)$ \\
\hline
\end{tabular}

*mean (SD)

Table 2 Incidence of foot drop $(n=68)$

\begin{tabular}{lll}
\hline & $\mathbf{n}(\%)$ & Incidence(95\% CI) \\
\hline Foot drop & & \\
None & $60(88.2)$ & \\
Yes & $8(11.8)$ & $11.8 \%(3.9 \%, 19.6 \%)$ \\
\hline
\end{tabular}

Table 3 Duration of recovery $(n=7)$

\begin{tabular}{lll}
\hline Variable & Median (IQR) & Minimum, Maximum \\
\hline $\begin{array}{l}\text { Duration of recovery } \\
\text { (month) }\end{array}$ & $3.0(5.0)$ & $2.0,11.0$ \\
\hline
\end{tabular}

\section{Discussion}

\section{Patient and demographic characeristics}

Facture that involves posterior aspect of acetabulum can be either in the form of posterior column fracture or posterior wall fracture. Posterior wall fracture of acetabulum is the most common and it accounts for $25-30 \%$ of all acetabular fractures. It is classified by Judet and Letournel ${ }^{1}$ under elementary type of fracture which is similar to posterior column fracture. ${ }^{2}$ This type of fracture most commonly occurs in young patients with high energy type of trauma such as motor vehicle accident. The majority of acetabular fractures are caused by some type of high-energy event, such as a car collision. Many times patients will have additional injuries that require immediate treatment.

In a smaller number of cases, a low-energy incident, such as a fall from standing, may cause an acetabular fracture in an older person who has weaker bones. In our study the mean age of patients that suffered from posterior wall and posterior column injury to the acetabulum was 38.64, with SD 16.17. This young age was attributed to the fact that these injuries were mostly due to high energy motor vehicle accident that involved motorcyclists which has the main bulk of younger population.

The average age mentioned in the literature was around 39 years. ${ }^{11}$ According to Malaysian demographic data in 2016, Malaysian with age 
group between 15 to 64 year-old formed the largest age group which was $69.5 \%$ and this contributes significantly to the high percentage of involvement for motorcyclists in this age group.

Male patients form the largest number of patients involved in this type of injury which is $82.4 \%$ while the percentage of female patients was $16.2 \%$. According to 2016 Malaysian demographic data, the ratio of male to female was 107 males for every 100 females which show that the ratio is almost equal to $1: 1$. Thus the higher number of male patients involved in our study was not because of the inequality in the number of male and female population but rather because of the differences in the number of male motorcyclists and female motorcyclists in Malaysia. Majority of the young motorcyclists were males which contributed much to the number of casualties in motor vehicle accident. 54 out of $67(79.4 \%)$ ) patients who suffered posterior wall and posterior column injury in this study were Malay followed by Chinese, 7 out of 67 , and Indian, 6 out of 67. This is mainly due to the fact that Malay population has the majority number in our demographic pattern, which forms $50.1 \%$ according to 2017 Malaysia demographic profile, with a number of them having low to moderate social-economic status that makes them prone to use cheaper method of transportation to perform their daily commute to work such as motorcycle.

There are 3 patterns of injury found in this study that involved posterior aspect of acetabulum. These are posterior wall fracture, posterior column fracture and transverse fracture. They were all categorized under elementary type of fracture of acetabulum. ${ }^{2} \mathrm{We}$ found out that the most common type of fracture was posterior wall fracture which accounts for $41.2 \%$. It was followed in this study by posterior column fracture ranging from $25 \%$ to $27 \%$ of incidence. Transverse fracture accounts for $1.5 \%$ to $4.4 \%$ of incidence. This is in line with the fact that posterior wall fractures are the most common type of acetabular fracture and account for approximately $24 \%$ of all acetabular fractures. ${ }^{12}$

In our study also we found out that the right acetabulum affected much more common (60.3\%) compared to the left side. This finding contradicts to study done by Maia et al. ${ }^{13}$ which in their study they found out that the left acetabulum affected much more common in $57.7 \%$ of their patients and only $42.3 \%$ of their patients had right ace tabular fracture. ${ }^{13}$ The sample size of their study was almost equivalent to our study which was 69 patients compared to 67 in ours.

In our study the right acetabulum is affected much more common than the left can be explained by the fact that our road system is of right hand drive system in which the right lower limb is exposed to the oncoming traffic much more readily compared to left lower limb. In the event of head on collision among the motorcyclists, the flexed right knee has the highest tendency to receive the impact first will lead to posterior hip dislocation and subsequently results in posterior wall fracture of right sided acetabulum. This explained why posterior wall fracture was the most common type of acetabular fracture and it explained also why the right acetabulum is much more commonly affected in our study. Transverse fracture type is the least common type of ace tabular fracture in our study mainly due to the fact that it requires direct blow to greater trochanter resulting in this pattern of ace tabular fracture. Direct blow to the hip is a rare type of mechanism of injury.
All of the patients were treated operatively with reconstruction plate through posterior approach using Kocher Lagen back method of approaching the hip from posterior. Majority of the approach were performed on the right side since a larger number of patients in this study sustained acetabular fracture on the right than on the left side. All of the patients were positioned according to standard positioning method in Kocher Lagen back approach, which is, lateral decubitus.

Among the entire 68 study group that were involved, only 8 participants had sciatic nerve palsy in the form of foot drop. From the study participants, the incidence of foot drop in the population was estimated to be $11.8 \%$ (95\% CI: 3.9\%, 19.6\%). Out of those who had sciatic nerve neuropraxia, 7 out of 8 patients $(87.5 \%)$ recovered. Among these patients, the minimum recovery time was 2.0 months and the maximum recovery time was 11.0 months. One patient did not achieve recovery until the end of this study.

Several simple precautions to prevent iatrogenic sciatic nerve injury can be practiced such as recommended by Letournel and Judet ${ }^{1}$ when operating, to keep the hip extended and the knee flexed to minimize traction neuropraxia to the sciatic nerve. They also recommended the use of transcondylar traction to maintain this position during the operation which they note reduced their postoperative sciatic nerve palsy rate from $18.4 \%$ to $3.3 \%$. Excessive retraction posteriorly with the hip in flexion or levering retractors placed in the lesser sciatic notch can place tension on or directly compress the sciatic nerve, respectively, and should be avoided. These traction neuropraxia can be minimized or avoided with the use of special type of retractor such as Hohman-type retractors with curved edges. The obturator internus tendon and muscle belly should be held between the nerve and the retractor.

\section{Conclusion}

Facture that involves posterior aspect of acetabulum can be either in the form of posterior column fracture or posterior wall fracture and transverse fracture. Posterior wall fracture of acetabulum is the most common and it accounts for $25-30 \%$ of all acetabular fractures. Effective management of these injuries requires a clear history of the temporal relationship between onset of symptoms and acetabular trauma or reconstructive surgery and possible pre-existing spinal disorders. Appropriate imaging studies, including plain radiographs, $\mathrm{CT}$, and MRI, and electro-my graphic studies can help localize the site of nerve injury. Of all, to be an iatrogenic injury should be prevented by taking little important measure preoperatively, intraoperative as well as close monitoring post operatively. Several important steps such as keeping the hip extended and the knee flexed will minimized traction neuropraxia. Excessive retraction posteriorly with the hip in flexion should be avoided as it causes tension and direct compression force on sciatic nerve. Similar consequences noted if levering retractors placed in the lesser sciatic notch. Extra caution to be taken while drilling or placing screws in the vicinity of the greater sciatic notch, appropriate fluoroscopic views should be obtained immediately to confirm safe instrument positioning. It is possible to have sciatic nerve palsy with reconstruction plate fixation of posterior wall of acetabulum through posterior approach but its incidence is quite low rather rare with experience.

\section{Acknowledgements}

None. 


\section{Conflict of interest}

Author declares that there is no conflict of interest.

\section{References}

1. Judet R, Judet J, Letournel E. Fractures of the Acetabulum: Classification and Surgical Approaches for Open Reduction. Preliminary Report. J Bone Joint Surg Am. 1964;46:1615-1646.

2. Letournel E. Acetabulum fractures: classification and management. Clin Orthop Relat Res. 1980;(151):81-106.

3. Helfet DL, Schmeling GJ. Somatosensory evoked potential monitoring in the surgical treatment of acute, displaced acetabular fractures. Results of a prospective study. Clin Orthop Relat Res. 1994;(301):213-220.

4. Haidukewych GJ, Scaduto J, Herscovici D Jr, et al. Iatrogenic nerve injury in acetabular fracture surgery: a comparison of monitored and unmonitored procedures. J Orthop Trauma. 2002;16(5):297-301.

5. Butt AJ, T McCarthy, Kelly IP, et al. Sciatic nerve palsy secondary to postoperative haematoma in primary total hip replacement. $J$ Bone Joint Surg Br. 2005;87(11):1465-1467.

6. Beauchesne RP, Schutzer SF. Myositis ossificans of the piriformis muscle: an unusual cause of piriformis syndrome. A case report. J Bone Joint Surg Am. 1997;79(6):906-910.
7. Derian PS, Bibighaus AJ. Sciatic nerve entrapment by ectopic bone after posterior fracture-dislocation of the hip. South Med J. 1974;67(2):209-210.

8. Schmalzried TP, Noordin S. Update on nerve palsy associated with total hip replacement. Clin Orthop Relat Res. 1997;(344):188-206.

9. Pekkarinen J, Alho A. Recovery of sciatic nerve injuries in association with total hip arthroplasty in 27 patients. $J$ Arthroplasty. 1999;14(3):305-311.

10. Schmalzried TP, Amstutz HC, Dorey FJ. Nerve palsy associated with total hip replacement. Risk factors and prognosis. J Bone Joint Surg Am. 1991;73(7):1074-1080.

11. Shrestha D, Dhoju D, Shrestha R, et al. Acetabular Fracture: Retrospective Analysis of Thirty Three Consecutive Cases with Operative Management. Kathmandu Univ Med J. 2014;12(48):279-287.

12. Giannoudis PV, Grotz MR, Papakostidis C, et al. Operative treatment of displaced fractures of the acetabulum. A meta-analysis. J Bone Joint Surg Br. $2005 ; 87(1): 2-9$.

13. Maia MS, Santos DC. Epidemiological Analysis on Acetabular Fractures. Rev Bras Ortop. 2011;46(1):23-26. 